\title{
Survey of Topological Insulators 2-3 Dimensional
}

\author{
Meri Algarni \\ Department of Physics \\ School of Arts and Science \\ Clark Atlanta University, Atlanta, GA, USA
}

\begin{abstract}
Topological separators are electronic materials that have a mass band crevice like a standard protector, yet have ensured directing states on their edge or surface. These states are conceivable because of the blend of turn circle connections and time inversion symmetry. The $2 \mathrm{D}$ topological cover is a quantum turn Hall encasing, which is a nearby cousin of the whole number quantum Hall state. A 3D topological protector bolsters novel turn enraptured 2D Dirac fermions on its surface. In this Colloquium article, we will audit the hypothetical establishment for topological encasings and superconductors and portray late tests in which the marks of topological separators have been watched. We will depict transport probes $\mathrm{HgTe} / \mathrm{CdTe}$ quantum wells that show the presence of the edge states anticipated for the quantum turn Hall protector. We will then talk about analyses on Bi1-xSbx, Bi2Se3, Bi2Te3 and Sb2Te3 that build up these materials as 3D topological separators and specifically test the topology of their surface states. We will then depict extraordinary states that can happen at the surface of a 3D topological encasing because of an initiated vitality crevice. An attractive crevice prompts a novel quantum Hall express that offers ascend to a topological magnetoelectric impact. A superconducting vitality hole prompts an express that backings Majorana fermions, and may give another setting to acknowledging proposition for topological quantum calculation. We will close by examining prospects for watching these outlandish states and also other potential gadget utilizations of topological protectors
\end{abstract}

\section{General Terms}

Topological Insulators

\section{Keywords}

Topological Insulator, two dimensions, three dimensions, Quantum Spin Hall Insulator (QSH), Graphene, Dirac electrons and Haldane model

\section{INTRODUCTION}

A repeating topic in consolidated matter material science has been the disclosure and order of unmistakable stages of matter. Regularly, stages can be comprehended utilizing Landau's approach, which describes states regarding hidden symmetries that are suddenly broken. During recent years, the investigation of the quantum Hall impact has prompted an alternate order worldview, based on the thought of topological request (Thouless, et al., 1982; Wen, 1995). The state in charge of the quantum Hall impact does not break any symmetries, but rather it characterizes a topological stage as in certain major properties, (for example, the quantized estimation of the Hall conductance, also, the quantities of gapless limit modes) are inhumane to smooth changes in materials parameters also, cannot change unless the framework goes through a quantum stage move.
In the previous five years another field has developed in dense matter material science, in view of the acknowledgment that the turn circle collaboration can prompt topological protecting electronic stages and on the expectation and perception of these stages in genuine materials (Bernevig, Hughes and Zhang, 2006; Fu and Kane, 2007; K"onig, et al., 2007; Hsieh, et al., 2008; Xia, et al., 2009a; Zhang, H., et al., 2009). A topological cover, like a customary encasing, has a mass vitality crevice isolating the most noteworthy involved electronic band from the least exhaust band. The surface (or edge in two measurements) of a topological cover, be that as it may, fundamentally has gapless states that are ensured by time inversion symmetry. The topological cover is firmly identified with the two-dimensional (2D) whole number quantum Hall state, which additionally has special edge states. The surface (or edge) conditions of a topological cover prompt a leading state with properties dissimilar to some other known 1D or 2D electronic frameworks. Notwithstanding their key intrigue, these states are anticipated to have unique properties that could be valuable for applications going from spintronic to the quantum calculation.

The idea of the topological request (Wen, 1995) is frequently used to portray the complicatedly related partial quantum Hall states (Tsui, Stormer, and Gossard, 1982), which require a naturally many body ways to deal with comprehend (Laughlin, 1983). In any case, topological contemplations additionally apply to the less difficult whole number quantum Hall states (Thouless, et al., 1982), for which a sufficient portrayal can be planned as far as single molecule quantum mechanics. In such manner, topological separators are like the number quantum Hall impact. Because of the nearness of a solitary molecule vitality hole, electron associations don't change the state in a fundamental way. Topological separators can be comprehended inside the structure of the band hypothesis of solids (Bloch, 1929). It is wonderful that after over 80 years, there are still fortunes to be revealed inside band hypothesis.

In this colloquium article, we will audit the hypothetical and exploratory establishments of this quickly creating field. We start in Section II with a prolog to topological band hypothesis, in which we will clarify the topological request in the quantum Hall impact and topological encasings. We will likewise give a short prolog to topological superconductors, which can be comprehended inside a comparable structure. A binding together component of these states is the mass limit correspondence, which relates the topological structure of mass precious stone to the nearness of gapless limit modes. Segment III will portray the 2D topological separator, otherwise called a quantum turn Corridor separator and talk about the disclosure of this stage in $\mathrm{HgCdTe}$ quantum wells. Segment IV is dedicated to 3D topological separators. We will survey their trial revelation in Bil-xSbx and later work on "second era" materials Bi2Se3 and Bi2Te3. Area V will concentrate on intriguing states that can happen at the surface 
of a topological separator because of an actuated vitality crevice. A vitality crevice incited by an attractive field or vicinity to an attractive material prompts a novel quantum Hall state, alongside a topological magnetoelectric impact. A vitality crevice because of nearness with a superconductor prompts an express that backings Majorana fermions, and may give another setting to acknowledging recommendations for topological quantum calculation. In Section VI we will close with a talk on new materials, new investigations and open issues.

\section{TOPOLOGICAL BAND THEORY 2.1 The Protecting State}

The protecting state is an essential condition of matter. The most straightforward encasing is a nuclear separator, with electrons bound to particles in shut shells. Such a material is electrically dormant because it takes a limited vitality to remove an electron. More grounded communication between particles in a gem prompts covalent holding. One of the triumphs of quantum mechanics in the twentieth century was the advancement of the band hypothesis of solids, which gives a dialect to depicting the electronic structure of such states. This hypothesis misuses the translational symmetry of the precious stone to characterize electronic states as far as their gem energy $\mathrm{k}$, characterized in an intermittent Brillouin zone. The Bloch States |um(k)I, characterized in a single unit cell of the gem, are eigenstates of the Bloch Hamiltonian $\mathrm{H}(\mathrm{k})$. The eigenvalues $E m(k)$ characterize vitality groups that overall frame the band structure. In a separator, a vitality crevice isolates the possessed valence band states from the vacant conduction band states. One can envision turning the Hamiltonian to insert ceaselessly between the two without shutting the vitality crevice. Such a procedure characterizes a topological proportionality between various protecting states. On the off chance that one receives a somewhat coarser "stable" topological grouping plan, which likens states with various quantities of inconsequential center groups, then all conventional separators are equal. Without a doubt, such separators are proportional to the vacuum, which as indicated by Dirac's relativistic quantum hypothesis additionally has a vitality crevice (for combine creation), a conduction band (electrons) and a valence band (positrons). Are all electronic states with a vitality hole topologically proportionate to the vacuum? The appropriate response is no, and the counterexamples are entrancing conditions of matter.

\subsection{2. Quantum Hall State}

The least complex counterexample is the whole number quantum Hall state (von Klitzing, Dorda, and Pepper, 1980; Prange and Girvin, 1987), which happens when electrons restricted to two measurements are set in a solid, attractive field.
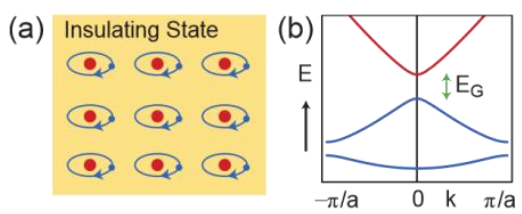

(d)

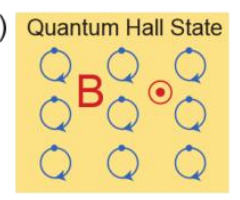

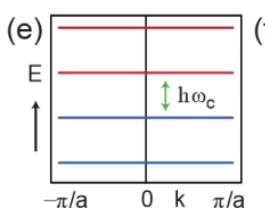

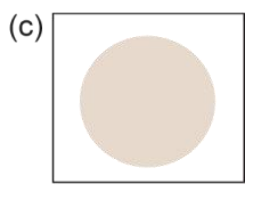

(f)

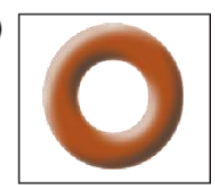

FIG. 1 (a, b, c) The protecting state. (a) delineates a nuclear protector, while (b) demonstrates a straightforward model protecting band structure. (d, e, f) The quantum Hall state. (d) delineates the cyclotron movement of electrons, and (e) demonstrates the Landau levels, which might be seen as a band structure. (c) and (f) demonstrate two surfaces which contrast in their sort, g. $g=0$ for the circle (c) and $g=1$ for the doughnut (f). The Chern number $n$ that recognizes the two states is a topological invariant like the variety.

The quantization of the electrons' roundabout circles with cyclotron recurrence $\omega c$ prompts quantized Landau levels with vitality 2). On the off chance that $\mathrm{N}$ Landau levels are filled and the rest are void, then a vitality hole isolates the involved and discharge states simply like in an encasing. Not at all like a separator, however, an electric field causes the cyclotron circles to float, prompting a Hall current described by the quantized Hall conductivity

$\sigma x y=\mathrm{Ne} 2 / \mathrm{h}$.

The quantization of $\sigma x y$ has been measured to one section in 109 (von Klitzing, 2005). This accuracy is a sign of the topological way of $\sigma x y$. Landau levels can be a "band structure." Since the generators of interpretations don't drive with each other in an attractive field, electronic states cannot be marked with energy. Be that as it may, if a unit cell with range $2 \pi \sim \mathrm{c} / \mathrm{eB}$ encasing a flux quantum is characterized, then cross section interpretations do drive, so Bloch's hypothesis permits states to be named by $2 \mathrm{D}$ precious stone energy $\mathrm{k}$. Without an occasional potential, the vitality levels are just the $\mathrm{k}$ autonomous Landau levels. Within sight of an occasional potential with a similar cross section periodicity, the vitality levels will scatter with $\mathrm{k}$. This prompts a band structure that seems to be indistinguishable to that of a typical encasing.

\subsection{Graphene and Dirac Electronic (GDE)}

A basic case of the quantum Hall impact in a band hypothesis is given by a model of graphene in an occasional attractive field presented by Haldane (1988). We will quickly deviate here to present graphene because it will give understanding into the origination of the 2D quantum turn Hall encasing and because the material science of Dirac electrons exhibits in graphene has essential parallels at the surface of a 3D topological separator. Graphene is a 2D type of carbon that is of high current intrigue. The least complex portrayal of graphene utilizes a two band display for the pz orbitals on the two comparable iotas in the unit cell of graphene's honeycomb grid. The Bloch Hamiltonian is then a $2 \times 2$ network,

$\mathrm{H}(\mathrm{k})=\mathrm{h}(\mathrm{k}) \cdot \sim \sigma$,

where $\sim \sigma=(\sigma \mathrm{x}, \sigma \mathrm{y}, \sigma \mathrm{z})$ are Pauli lattices and $\mathrm{h}(\mathrm{k})=$ $(\mathrm{hx}(\mathrm{k}), \mathrm{hy}(\mathrm{k}), 0)$. The blend of reversal $(\mathrm{P})$ and time inversion ( $\mathrm{T}$ ) symmetry requires $\mathrm{hz}(\mathrm{k})=0$. In graphene, they happen at two focuses, $\mathrm{K}$ and $\mathrm{K} 0=-\mathrm{K}$, whose areas at the Brillouin zone corners are settled by graphene's rotational symmetry. For little $\mathrm{q} \equiv \mathrm{k}-\mathrm{K}, \mathrm{h}(\mathrm{q})=\sim \mathrm{vFq}$, where $\mathrm{vF}$ is speed, so $\mathrm{H}(\mathrm{q})=$ $\sim \mathrm{vFq} \sim \sigma$ has the type of a $2 \mathrm{D}$ massless Dirac Hamiltonian.

$\mathrm{P}$ and $\mathrm{T}$ symmetry secure the decline at the Dirac point. By breaking these symmetries, the decadence can be lifted. For example, P-symmetry is disregarded if the two iotas in the unit cell are inequivalent. This permits $\mathrm{hz}(\mathrm{k})$ to be non-zero. If $\mathrm{hz}(\mathrm{k})$ is little, then close $\mathrm{K}$ (3) turns into a huge Dirac Hamiltonian,

$\mathrm{H}(\mathrm{q})=\sim \mathrm{vFq} \cdot \sim \sigma+\mathrm{m} \sigma \mathrm{z}$ 
where

$$
\begin{array}{ll}
\mathrm{m} & =\quad \mathrm{hz}(\mathrm{K}) . \quad \text { The dispersion } \\
\mathrm{E}(\mathrm{q}) & = \pm \mathrm{p}|\sim \mathrm{vFq}| 2+\mathrm{m} 2
\end{array}
$$

Take note of that T-symmetry requires the Dirac point at K0 has a mass $\mathrm{m} 0=\mathrm{hz}(\mathrm{K} 0)$ with a similar greatness and sign, $\mathrm{m} 0$ $=\mathrm{m}$. This state portrays a standard separator. Haldane (1988) envisioned lifting the decadence by breaking $\mathrm{T}$ symmetry with an attractive field that is zero on the normal, yet has the full symmetry the cross section. This bother permits nonzero $\mathrm{hz}(\mathrm{k})$ and acquaints a mass with the Dirac focuses. Be that as it may, $\mathrm{P}$ symmetry requires the masses at $\mathrm{K}$ and $\mathrm{K} 0$ have an opposite sign, $\mathrm{m} 0=-\mathrm{m}$. Haldane demonstrated this gapped state is not a separator, yet rather a quantum Hall state with $\sigma x y=\mathrm{e} 2 / \mathrm{h}$.

This non-zero Hall conductivity can be comprehended as far as (2). For a two-level Hamiltonian of the type of (3) it is outstanding that the Berry flux (Berry, 1984) is identified with the strong edge subtended by the unit vector. This tallies the quantity of times $\mathrm{h}^{\wedge}(\mathrm{k})$ wraps around the unit circle as a component of $\mathrm{k}$. At the point when the masses $\mathrm{m}=\mathrm{m} 0=0$ $\mathrm{h}^{\wedge}(\mathrm{k})$ is bound to the equator $\mathrm{hz}=0$, with a unit (and inverse) twisting around each of the Dirac, focuses where $|h|=0$. For little yet limited $\mathrm{m},|\mathrm{h}| 6=0$ all over the place, and $\mathrm{h}^{\wedge}(\mathrm{K})$ visit the north or south shaft, contingent upon the indication of $\mathrm{m}$. It takes after that every Dirac point contributes $\pm \mathrm{e} 2 / 2 \mathrm{~h}$ to $\sigma x y$. In the protecting state with $\mathrm{m}=\mathrm{m} 0$ the two drop, so $\sigma \mathrm{xy}=0$. In the Quantum Hall State, they include.

It is fundamental that there was a considerable number of Dirac focuses since the Hall conductivity would be quantized to a whole half number. This is ensured by the fermion multiplying hypothesis (Nielssen and Ninomiya, 1983), which expresses that for a T-invariant framework Dirac focuses must come in sets. We will come back to this issue in area IV, where the surface of a topological protector gives an escape clause to this hypothesis.

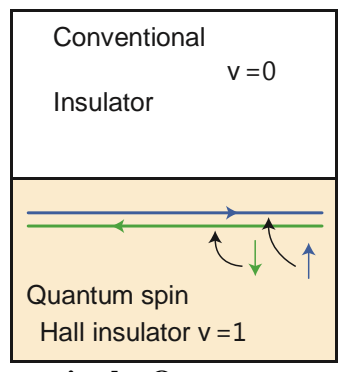

FIG. 2 Edge States in the Quantum turn Hall Encasing Demonstrates the Interface between a QSHI and a Standard Protector

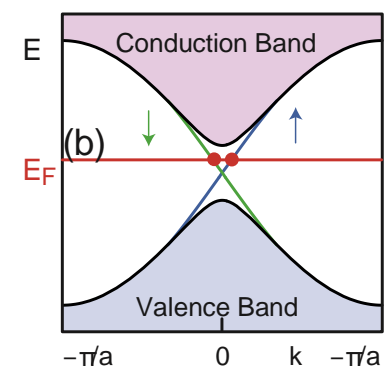

FIG. 3 Demonstrates the Edge State Scattering in the Graphene Show, in which here and there Twists Engender in Inverse Headings
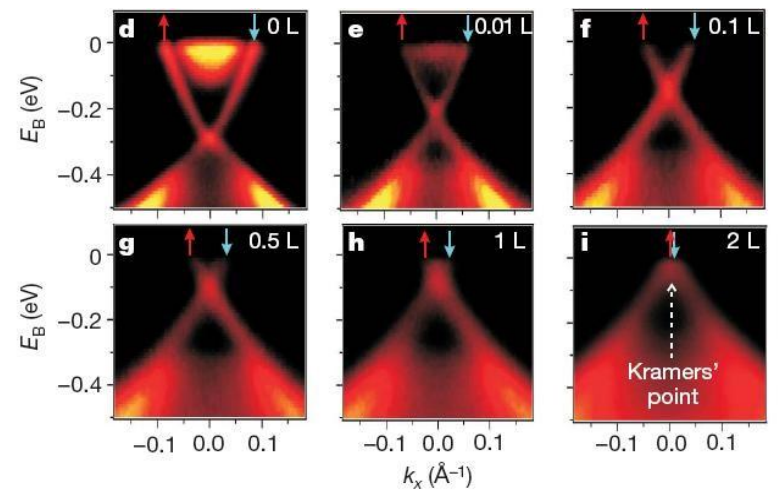

FIG 4. Bi2 $\mathrm{Se} 3 \mathrm{Control} \mathrm{E}_{\mathrm{F}}$ on surface by only a single Dirac point exposing to $\mathrm{NO}_{2}$.

\section{CONCLUSION}

Despite the fact that the essential properties of topological covers have been built up, the field is at an early stage in its improvement. There is much work to be done to understand the capability of these new and intriguing materials. In this finishing up area, we will talk about some exceptionally late advancements and look toward what's to come.

In the historical backdrop of consolidated matter physical science, the absolute most imperative fixing in the rise of another field is the flawlessness of the methods for creating great materials. For instance, the perplexing material science of the partial quantum Hall impact could never have risen without ultra-high portability GaAs. Topological protector materials should be idealized so that they protect. There has been a significant advance toward this path. For example, transport investigates $\mathrm{Ca}$-doped gems of $\mathrm{Bi} 2 \mathrm{Se} 3$ show explicit protecting conduct beneath around $100 \circ \mathrm{K}$ (Checkelsky, et al., 2009). Nonetheless, the electrical resistance immerses at low temperature, and the surface streams give off an impression of being overpowered by either mass ebbs and flows or ebbs and flow in a layer close to the surface. This is a testing issue since tight crevice semiconductors are extremely delicate to doping. In any case, it appears to be confident that there is sufficient opportunity to get better. Thin movies created, for example, by mechanical peeling (as in graphene), or chemically produced Bi2Se3 strips and wires (Peng, et al., 2010) might be useful in such manner. An especially encouraging new bearing is the development of epitaxial movies of Bi2Se3. This has prompted the current perception of Landau quantization in the Dirac surface states (Cheng, et al., 2010; Hanaguri, et al., 2010). It will dampen enthusiasm to watch such quantization in a vehicle contemplate. Exhibit materials represent a test because of rivalry amongst surface and mass states (Checkelsky, et al., 2009; Taskin and Ando, 2009). The point by point investigation of the electronic and turn transport properties of the surface states is called for, supplemented by a large group of different tests, extending from optics to burrowing spectroscopy.

Another bearing for future advancement will be the investigation of heterostructures including topological protectors and different materials. Notwithstanding giving way to securing and controlling the number of inhabitants in the surface states, such structures could give a stage towards the more extended term objective of designing intriguing States, for example, Majorana fermions, with the surface states. There are numerous materials issues to be explained so as to discover fitting attractive and superconducting materials 
which show the suitable vicinity impacts with the surface states, and point by point examinations will be important to portray those states. An energizing late improvement like this is the revelation of superconductivity in $\mathrm{Cu}$-doped $\mathrm{Bi} 2 \mathrm{Se} 3$ (Hor, et al., 2010a). One can envision gadgets created with strategies of tweak doping that have demonstrated amazingly effective in semiconductor material science. One can likewise envision different gadgets that incorporate beautiful materials with topological protectors, to exploit the uncommon turn properties of the surface states.

Topological protecting conduct is probably going to emerge in different classes of materials, notwithstanding the paired mixes $\mathrm{Bi} 1-\mathrm{xSbx}, \mathrm{Bi} 2 \mathrm{Te} 3, \mathrm{Bi} 2 \mathrm{Se} 3$, and $\mathrm{Sb} 2 \mathrm{Te} 3$. If one extends one's skyline to ternary mixes (or past) the likely outcomes for colorful materials increase. Competitor materials will be restricted hole semiconductors which incorporate substantial components. One fascinating class of materials are move metal oxides including iridium. Shitade, et al. (2009) have anticipated that $\mathrm{Na} 2 \mathrm{IrO} 3$ is a feeble topological separator. Pesin and Balents (2010) have proposed that specific iridium-based pyrochlore mixes might be solid topological encasings. Since these materials included electrons, an essential issue will be to comprehend the interchange between solid electron-electron cooperations and the turn circle association. Extremely late hypothetical work predicts topological cover conduct in ternary Heusler compounds (Chadov, et al., 2010; Lin, et al., 2010a) and different materials (Lin, et al., 2010b,c; Yan, et al., 2010). These are energizing new headings where facilitate hypothetical, and trial work is called for.

Topological superconductors introduce another energizing outskirt heading. Notwithstanding watching the surface Majorana modes anticipated for $3 \mathrm{He} \mathrm{B}$ (Chung and Zhang, 2009), it will be exceptionally fascinating to foresee and watch electronic topological superconductors, to portray their surface modes and to investigate their potential utility. To this end, there has been a late advance in creating techniques to hypothetically distinguish topological superconductors considering their band structure (Fu and Berg, 2009; Qi, Hughes and Zhang, 2010).

For the most part, there might be other fascinating associated states identified with topological protectors and superconductors. For instance, Levin and Stern (2009) demonstrated that a T-invariant fragmentary quantum turn Hall state could be topologically steady. This focuses on a general hypothetical issue related to the topological arrangement of associating frameworks: How would we bind together the apparently different thoughts of topological request typified by Thouless, et al. (1982) and by Wen (1995)? The topological field hypotheses examined by Qi, Hughes, and Zhang (2008), and additionally tempting associations with string hypothesis (Ryu and Takayanagi, 2010) give ventures toward this path. However, an entire hypothesis stays to be produced.

To close, the current advances in the material science of topological protectors have been driven by a rich exchange between hypothetical knowledge and trial revelations. There is the explanation behind the good faith that this field will keep on developing in energizing new bearings.

\section{ACKNOWLEDGMENTS}

The author would like to think Saudi Arabian Cultural Mission (SACM) for the financially support through Al-Baha University.

\section{REFERENCES}

[1] Haldane, F. D. M., 1988, Phys. Rev. Lett. 61, 2015.

[2] Qi, X. L., R. Li, J. Zang and S. C. Zhang, 2009, Science $323,1184$.

[3] Levin, M. and A. Stern, 2009, Phys. Rev. Lett. 103, 196803.

[4] Pesin, D. A., and L. Balents, 2010, Nat. Phys. 6, 376.

[5] Fradkin E., E. Dagotto, and D. Boyanovsky, 1986, Phys. Rev. Lett. 57, 2967.

[6] Ivanov, D. A., 2001, Phys. Rev. Lett. 86, 268.

[7] Jackiw, R., and P. Rossi, 1981, Nucl. Phys. B, 190681.

[8] Park, S. R., W. S. Jung, C. Kim, D. J. Song, C. Kim, S. Kimura, K. D. Lee and N. Hur, 2010, Phys. Rev. B 81, 041405(R).

[9] Read, N. and D. Green, 2000, Phys. Rev. B 61, 10267.

[10] Bernevig, B. A., T. A. Hughes, and S. C. Zhang, 2006, Science 314, 1757.

[11] Das Sarma, S., C. Nayak, and S. Tewari, 2006, Phys. Rev. B 73, 220502(R).

[12] Dolev, M., M. Heiblum, V. Umansky, A. Stern, and D. Mahalu, 2008, Nature 452, 829. 